\title{
Identification of three cytotoxic early proteins of mycobacteriophage L5 leading to growth inhibition in Mycobacterium smegmatis \\ Correspondence \\ Jan Rybniker \\ jan.rybniker@uk-koeln.de \\ Received 25 January 2008 \\ Revised 16 April 2008 \\ Accepted 1 May 2008

\author{
Jan Rybniker, ${ }^{1}$ Georg Plum, ${ }^{2}$ Nirmal Robinson, ${ }^{2}$ Pamela L. Small ${ }^{3}$ \\ and Pia Hartmann ${ }^{1,4}$ \\ ${ }^{1}$ 1st Department of Internal Medicine, University of Cologne, 50924 Cologne, Germany \\ ${ }^{2}$ Institute for Medical Microbiology, Immunology and Hygiene, University of Cologne, 50924 \\ Cologne, Germany \\ ${ }^{3}$ Department of Microbiology, University of Tennessee, Knoxville, TN 37996, USA \\ ${ }^{4}$ Department of Internal Medicine 1, Division of Infectious Diseases, University of Regensburg, \\ 93042 Regensburg, Germany
}

\begin{abstract}
Mycobacteriophage L5 is a temperate phage with a broad host range among the fast- and slowgrowing mycobacteria such as Mycobacterium smegmatis, Mycobacterium tuberculosis, Mycobacterium avium and Mycobacterium ulcerans. L5 switches off host protein synthesis during the early stage of lytic growth, as was previously shown by protein expression profiling. Also, lethal genetic elements have been identified in L5 based on the fact that transformants could not be obtained with these genes. Using an inducible mycobacterial shuttle vector, we have identified three ORFs within an early operon of mycobacteriophage L5 which encode gene products (gp) toxic to the host M. smegmatis when expressed. These ORFs, coding for gp77, gp78 and gp79, presumably function as shut-off genes during early stages of phage replication. There is evidence that cell division is affected by one of the proteins (gp79). The transcription of the cytotoxic polypeptides is directed by a promoter situated in ORF83 and transcription control is achieved through the phage repressor gp71, which is shown by co-expression of this protein. The findings presented here should provide useful tools for the molecular genetics of mycobacteria. Further analysis of these and other mycobacteriophage-derived toxic polypeptides, together with the identification of their cellular targets, might provide a tool for the rapid identification of promising drug targets in emerging and re-emerging mycobacterial pathogens.
\end{abstract}

\section{INTRODUCTION}

To date more than 250 mycobacteriophages have been isolated and the genomic sequences of more than 30 phages are available in the NCBI database. Taken together, these relatively large genomes code for more than 3000 putative proteins, many of which are without a match in the database (Hatfull et al., 2006; Pedulla et al., 2003). The functions of the vast majority of these mycobacteriophage ORFs remain unknown and only a few proteins have been expressed and examined in detail. Even more striking is the genomic diversity of mycobacteriophages, and exchange of genes among the phages themselves and among their hosts has led to a vivid and probably highly influential evolutionary relationship between virus and bacterial host.

Extensive comparison of the mycobacteriophage genomes and amino acid sequences and their organization in

Abbreviations: gp, gene product; PBP, penicillin-binding protein. so-called 'phamilies' (putative proteins with amino acid identity of $25 \%$ or more) showed that almost half of the phage-derived sequences match non-phage and predominantly bacterial genes (Hatfull et al., 2006). Furthermore, some of these non-phage-associated genes are homologues of suspected mycobacterial virulence genes and genes that are involved in bacterial cell-wall synthesis (Pedulla et al., 2003). On the host's side, the sequencing of mycobacterial genomes led to the identification of many prophages, which presumably influence the phenotype and virulence of some mycobacteria (Stinear et al., 2007).

Mycobacteriophage L5, a temperate phage isolated from Mycobacterium smegmatis, was the first mycobacteriophage to be sequenced (Hatfull \& Sarkis, 1993). L5 forms stable lysogens in $M$. smegmatis and has a broad host range among the pathogenic mycobacteria (Rybniker et al., 2006). Although L5 is a temperate phage, the following evidence suggests that the phage switches off host protein 
synthesis during early lytic growth. First, a quantitative decrease of host proteins is seen minutes after induction of a thermo-inducible L5 lysogen (Hatfull \& Sarkis, 1993), and second, it has been reported that a genetic element spanning ORF83 to ORF77 does not transform in $M$. smegmatis (Donnelly-Wu et al., 1993). These ORFs are situated on the right arm of the L5 genome, which encodes primarily early regulatory proteins.

The potential presence of host shut-off proteins in the temperate phage L5 implies an extremely tight silencing of the genes responsible by the L5 repressor. The repressor encoded by ORF71 is responsible for the temperate phenotype and the superinfection immunity of lysogens. Transcriptional silencing is achieved by binding of the repressor protein within or downstream of the four early promoter sequences identified so far (Brown et al., 1997; Donnelly-Wu et al., 1993).

The host shut-off phenomenon is well known for many lytic bacteriophages as well as for viruses of the eukaryotic cell (Fenwick \& Clark, 1982; Sharma et al., 2004; Svenson \& Karlstrom, 1976). It has recently been shown that the systematic exploration of staphylococcal bacteriophage derived shut-off proteins and their targets in Staphylococcus aureus can lead to the identification of new antibiotic chemical compounds (Liu et al., 2004).

For the identification of phage-derived toxic proteins, the existence of tightly regulated inducible expression vectors is a prerequisite. The recent development of inducible mycobacterial shuttle plasmids carrying the acetamidase promoter of $M$. smegmatis enabled us to screen for potentially toxic ORFs within the regulatory region of mycobacteriophage L5 (Daugelat et al., 2003).

Candidate genes were amplified from the L5 genetic region that does not transform in M. smegmatis and were inserted in a set of modified acetamidase-inducible expression plasmids. Here we present data showing that the L5 gene products (gp) 77, 78 and 79 are toxic to its natural host. Furthermore, we provide new insight into the regulation of these proteins by the description of a new promoter element and transcription control through the L5 repressor gp71.

\section{METHODS}

Bacterial strains, bacteriophages and plamids. Details of the bacterial strains, phage and plasmids used in this study are given in Table 1. Since the pSD24 acetamidase promoter shows intrinsic activity in Escherichia coli, amplification of some plasmid constructs carrying toxic proteins was not possible. To address this problem, the two lac operator elements of plasmid pQE80 (Qiagen) were amplified using the primers lacOfor (5'-TTTTATCCATGGATCGTTGTGAGCGGATAA- $\left.3^{\prime}\right)$ and lacOrev (5'-TCTGCAGCTGGATCCGTGATGGTGATGGTG-3') and cloned between the acetamidase promoter and multiple cloning site of pSD24, giving pJR5 (Table 1, Fig. 1). This allowed the propagation of otherwise toxic plasmids in E. coli strains constitutively expressing the lac repressor such as Fusion-Blue (Clontech). Insertion of this DNA fragment had no influence on the function of the acetamidase promoter in $M$. smegmatis.

Growth conditions. M. smegmatis was grown using Middlebrook 7H10 agar or Middlebrook 7H9 broth (Difco) supplemented with $10 \%$ OADC (oleic acid, albumin fraction V, dextrose, catalase),

Table 1. Strains, bacteriophages and plasmids used in the study

See Fig. 2 and Fig. 5 for additional plasmids generated in this study.

\begin{tabular}{|c|c|c|}
\hline & Description & Reference or source \\
\hline Fusion blue & $\begin{array}{l}\text { endA1 hsdR17 }\left(\mathrm{r}_{\mathrm{K} 12}^{-} \mathrm{m}_{\mathrm{K} 12}^{+}\right) \text {supE44 thi-1 recA1 gyrA96 relA1 lac } \\
\mathrm{F}^{\prime}\left[\operatorname{proA}^{+} B^{+} \text {lacl }^{\mathrm{q}} Z \Delta \mathrm{M} 15:: \operatorname{Tn} 10\left(\mathrm{Tet}^{\mathrm{R}}\right)\right]\end{array}$ & Clontech \\
\hline $\mathrm{DH} 5 \alpha$ & $\begin{array}{l}\mathrm{F}^{-}, \phi 80 \mathrm{~d} l a c Z \Delta \mathrm{M} 15 \Delta(\text { lacZYA-argF }) \text { U169 deoR recA1 endA1 } \\
\text { hsdR17 }\left(\mathrm{r}_{\mathrm{k}}^{-} \mathrm{m}_{\mathrm{k}}^{+}\right) \text {phoA supE44 } \lambda^{-} \text {thi-1 gyrA96 relA1 }\end{array}$ & Lab collection \\
\hline M. smegmatis $\mathrm{mc}^{2} 155$ & High-efficiency transformation mutant & Snapper et al. (1990) \\
\hline Mycobacteriophage L5 & Temperate phage of $M$. smegmatis & \\
\hline \multicolumn{3}{|l|}{ Plasmids } \\
\hline pQE80L & Expression vector carrying the cis-laq $I^{\mathrm{q}}$ repressor gene & Qiagen \\
\hline pSD24 & Acetamide-inducible mycobacterial expression vector & Daugelat et al. (2003) \\
\hline pJR5 & $\begin{array}{l}\text { pSD24 carrying two lac operators between acetamidase promoter and } \\
\text { multiple cloning site }\end{array}$ & This study \\
\hline pJR6 & pJR7 with L5 repressor gp71 & This study \\
\hline pJR10 & pJR7 without acetamidase promoter (Fig. 1) & This study \\
\hline
\end{tabular}


$0.05 \%$ Tween 80 and $0.5 \%$, v/v, glycerol. E. coli strains were grown using LB agar or broth. Cells transformed with the derivatives of pSD24 were grown on medium containing $50 \mu \mathrm{g}$ hygromycin $\mathrm{B} \mathrm{ml}^{-1}$ (Roth) for M. smegmatis or $100 \mu \mathrm{g}$ hygromycin $\mathrm{B} \mathrm{m} \mathrm{m}^{-1}$ for E. coli. Ampicillin (Roth) was used at a final concentration of $100 \mu \mathrm{g} \mathrm{ml}^{-1}$ and tetracycline (Roth) at a concentration of $20 \mu \mathrm{g} \mathrm{ml}^{-1}$.

DNA manipulation. DNA manipulation, electrophoresis and PCRs were carried out using standard techniques (Sambrook \& Russell, 2001). All PCRs for subsequent cloning were performed using Phusion high fidelity polymerase (Finnzymes) according to the instructions provided by the manufacturer.

DNA cloning. All cloning steps were done by homologous recombination using the In-Fusion PCR cloning kit (Clontech). All primers had $15 \mathrm{bp}$ homology with each end of the linearized vector at their $5^{\prime}$-end followed by 15 bp specific sequence homologues to the L5-ORF amplified as recommended by the In-Fusion protocol. Primers for the amplification and cloning of ORF77 in pJR5 are given as examples: forward primer $5^{\prime}$-TCACCATCACGGATCCatgATCGACGGCAA-3', reverse primer 5'-TCTGCAGCTGGATCC tcaGCCCGCCAGCGC-3'; sequence with homology to the linearized vector is underlined whereas sequence with homology to ORF77 is shown in italics (atg, start codon; tca, stop codon). With regard to pSD24, pJR5, pJR6, pJR7, pJR8 and pJR10 the single BamHI restriction site was used for all insertional cloning steps with subsequent expression, whereas pQE80 was linearized with BamHI and HindIII. Sequence identity was confirmed for all clones by sequencing the first $250-500 \mathrm{bp}$ downstream of the restriction enzyme site that was used for the cloning procedure.

Electroporation of mycobacteria. Electrocompetent M. smegmatis cells were prepared by growing the bacteria in $7 \mathrm{H} 9$ broth (containing OADC and Tween 80) to an $\mathrm{OD}_{600}$ of 1.0. The cells were kept on ice for $90 \mathrm{~min}$ and harvested by centrifugation at $4000 \mathrm{~g}$ for $15 \mathrm{~min}$. The cells were washed three times in ice-cold $10 \%$ glycerol and resuspended in a $1 / 100$ volume of $10 \%$ glycerol. Settings for electroporation were $2.5 \mathrm{kV}, 25 \mu \mathrm{F}$ and a resistance of $200 \Omega$.

Identification of toxic phage ORFs. Fig. 2 illustrates the plasmids that were created for the identification of toxic phage ORFs. $M$. smegmatis clones were subcultured on $7 \mathrm{H} 10$ agar containing $0.1 \%$ acetamide (Sigma) and E. coli DH5 $\alpha$ clones on LB agar supplemented with $0.2 \mathrm{mM}$ IPTG (Roth). Clones showing growth inhibition on selective media were further examined using serial dilutions of acetamide for $M$. smegmatis and IPTG for E. coli followed by $\mathrm{OD}_{600}$ readings after $4 \mathrm{~h}$ for E. coli and after $12 \mathrm{~h}$ for M. smegmatis. Also, standard survival curves were created for the clones of interest (see Fig. 4). For survival curves and $\mathrm{OD}_{600}$ readings, subculturing of $M$. smegmatis clones with derivatives of pSD24 carrying toxic ORFs was kept to a minimum since pSD24 plasmid propagation is not fully stable over several subculturing steps.

Fluorescence microscopy. This was performed as described previously (Robinson et al., 2007). In brief, individual colonies were scraped off $7 \mathrm{H} 10$ agar plates and washed in $1 \times$ PBS. Then $180 \mu \mathrm{l}$ $0.1 \mathrm{M} \mathrm{NaHCO}_{3}$ and $20 \mu \mathrm{lFITC}\left(100 \mathrm{mg} \mathrm{ml}^{-1}\right.$; Sigma) were added to approximately $25 \mathrm{mg}$ bacteria (wet wt). After incubation for $30 \mathrm{~min}$ at $37{ }^{\circ} \mathrm{C}$ the stained cells were washed three times with PBS and mounted on a glass slide. Microscopy was done on an Olympus IX81 inverted fluorescence microscope.

Insertion of the repressor gene ORF71 in pJR5 and coexpression studies. A sequence spanning bp 44331 to bp 45045 of the L5 genome was amplified by PCR using the forward primer 5'
GCGCGGCCGCGGTACCTTAGGGCTCCGAGA-3' and the reverse primer $5^{\prime}$-CACTTCTTTATCTAGATAACGCCAGTCGAT-3'. This amplified sequence covers ORF71, which codes for the L5 repressor, and the two promoters $\mathrm{P} 1$ and $\mathrm{P} 2$, which are situated upstream of ORF71. The PCR product was cloned into pJR5 linearized with XbaI and Acc65I, giving pJR6 (Fig. 1).

A PCR product spanning ORF83 to ORF77 was cloned in pJR5 and pJR6 respectively (Fig. 2). Plasmid DNA was amplified in E. coli Fusion-Blue and $250 \mathrm{ng}$ of each construct was electroporated into $80 \mu$ l competent $M$. smegmatis cells. Transformants were plated onto $7 \mathrm{H} 10$ plates containing $50 \mu \mathrm{g}$ hygromycin $\mathrm{B} \mathrm{ml}{ }^{-1}$.

Generation of mycobacterial promoter-probe vectors and promoter identification. The lac $Z$ gene of pMC1871 was amplified by PCR using the primers pSDlacFO $\left(5^{\prime}\right.$-TTTTATCCATGGATCCGTCGTTTTACAACG-3') and pSDlacRE (5'-TCTGCAGCTGGATCTCCCCTGCCCGCTTAT-3'). Primer pSDlacRE deletes the second BamHI site, leaving only one functional BamHI site upstream of the lacZ gene. The PCR product was purified and cloned into BamHI-linearized pSD24, giving pJR7 (Fig. 1). The acetamidase promoter of pJR7 was deleted by digestion with BamHI and XbaI and subsequent religation of the vector ends using an oligo-linker made by annealing the oligos LinkXbaBamFO (5'-CTAGCCCGAGGTATCTAGATATCTTTG-3') and LinkXbaBamRE (5'-GATCCAAAGATATCTAGATACCTCGGG-3'), resulting in the plasmid pJR10 (Fig. 1). To generate pJR8, which is pJR7 constitutively expressing the L5 phage repressor gp71, a similar procedure was chosen as described for pJR5 and pJR6. These three plasmids, pJR7, pJR8 and pJR10, gave the basis for several constructs used for the identification of promoter activity in ORF83, which was amplified by PCR together with $12 \mathrm{bp}$ of the $5^{\prime}$-end of ORF82, leading to a fusion protein of the gene product of ORF82 and the $\beta$-galactosidase protein (see Fig. $5 \mathrm{a}$ ). For pJR7::8382 and pJR8::8382 the genetic element spanning ORF83, ORF82, the intergenic region of ORF82/ORF81 and the start codon of ORF81 was amplified and cloned in-frame with the lacZ gene. The $21 \mathrm{bp}$ sequence between the stop codon of ORF82 and the start codon of ORF81 contains a repressor-binding site (Brown et al., 1997).

Quantitative $\boldsymbol{\beta}$-galactosidase assays. $M$. smegmatis clones were grown to mid-exponential phase in $5 \mathrm{ml} 7 \mathrm{H} 9$ broth, the $\mathrm{OD}_{600}$ was measured and the cells were harvested and resuspended in $500 \mu \mathrm{l}$ cold PBS. As controls all constructs were grown in parallel in the presence of $0.1 \%$ acetamide for $6 \mathrm{~h}$. Glass beads $(0.1 \mathrm{~mm})$ were added and the cell suspension was disrupted by using a bead mill (MM200, Retsch) for a total of $15 \mathrm{~min}$ at maximum speed with $5 \mathrm{~min}$ on ice after every $5 \mathrm{~min}$ of disruption. After centrifugation for $5 \mathrm{~min}$ at $4{ }^{\circ} \mathrm{C}$ and $10000 \mathrm{~g}, \beta$-galactosidase activity was measured by adding $30 \mu \mathrm{l}$ supernatant to $210 \mu \mathrm{l}$ Z-buffer $\left(0.06 \mathrm{M} \mathrm{Na}_{2} \mathrm{HPO}_{4} .7 \mathrm{H}_{2} \mathrm{O}, 0.04 \mathrm{M}\right.$ $\mathrm{NaH}_{2} \mathrm{PO}_{4} \cdot \mathrm{H}_{2} \mathrm{O}, 0.01 \mathrm{M} \mathrm{KCl}, 0.001 \mathrm{M} \mathrm{MgSO}{ }_{4}, 0.05 \mathrm{M} \beta$-mercaptoethanol, $\mathrm{pH}$ 7.0). The reaction was started by the addition of $60 \mu \mathrm{l}$ ONPG (Sigma) at $4 \mathrm{mg} \mathrm{ml}^{-1}$ in $0.1 \mathrm{M}$ sodium phosphate $(\mathrm{pH} 7.5)$ and subsequently incubated at $37{ }^{\circ} \mathrm{C}$. After development of a visible colour change to yellow the reaction was stopped by the addition of $500 \mu \mathrm{l} 1 \mathrm{M} \mathrm{Na}_{2} \mathrm{CO}_{3}$ and $A_{420}$ was measured. $\beta$-Galactosidase activity was calculated in Miller units using the following equation: $1000 \times A_{420} /\left(t \times v \times \mathrm{OD}_{600}\right)$ with $t$ being the time from the addition of ONPG to the addition of $\mathrm{Na}_{2} \mathrm{CO}_{3}$ and $v$ the culture volume $(5 \mathrm{ml})$ (Zhang \& Bremer, 1995).

Primer extension. Five picomoles of primer Seclaq (5'TTGGGTAACGCCAGGGTTTTC-3') was end-labelled with $\left[\gamma^{32} \mathrm{P}\right]$ ATP using 20 units T4-polynucleotide kinase (Fermentas). Labelled primer was passed through a Sephadex-G50 NICK-column (GE-Healthcare) to remove unincorporated nucleotides. Total RNA was isolated from M. smegmatis clones carrying either pJR10:: 83 or 


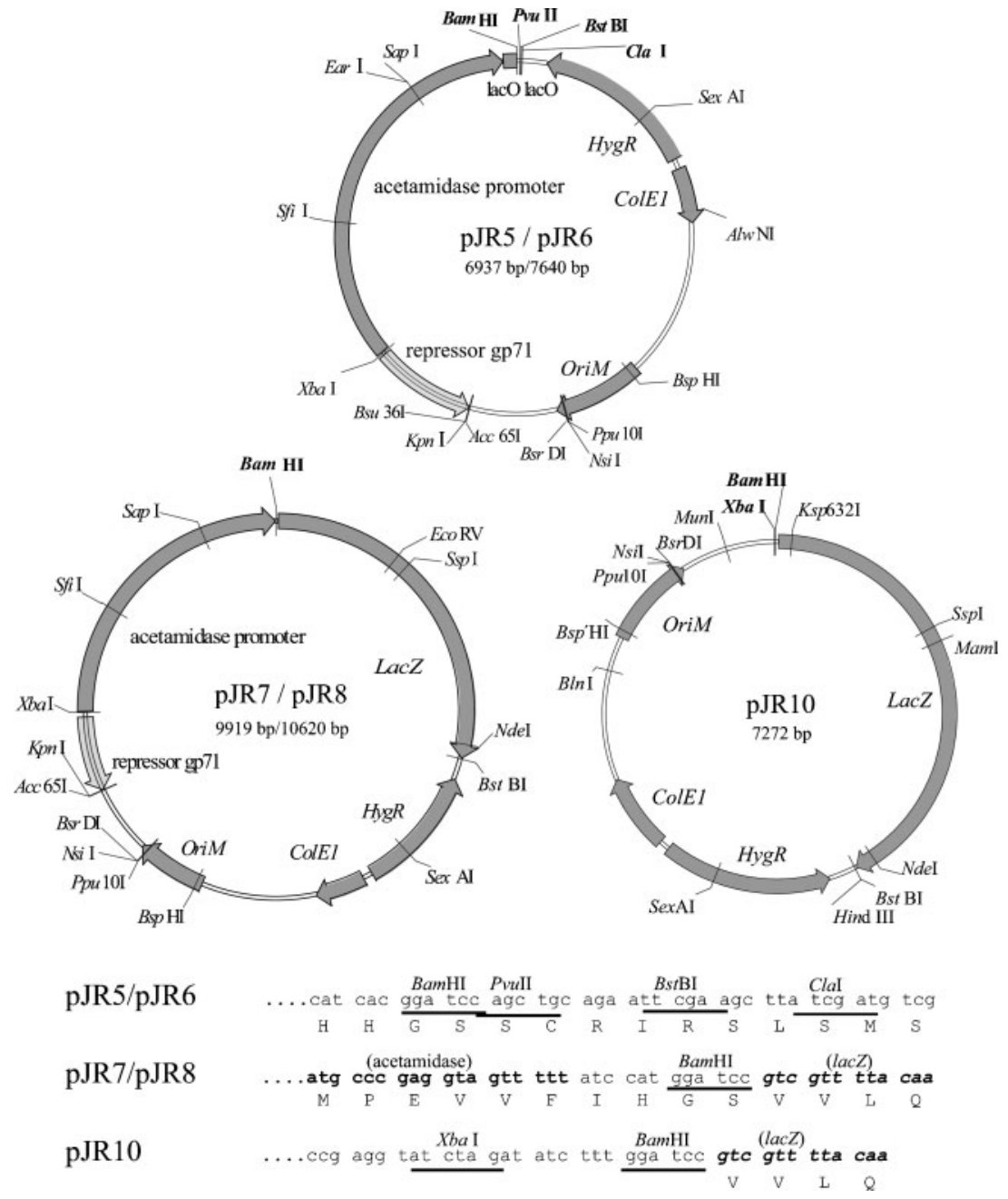

Fig. 1. Map of the modified acetamidase expression plasmid pJR5 and the new promoter-probe plasmids pJR7/pJR8 and pJR10. Only pJR6 and pJR8 carry the L5 repressor gene gp71 used for co-expression studies. The lower part shows the multiple cloning sites.
pJR10 as a control. Five micrograms of total RNA was incubated with approximately $50 \mathrm{fmol}$ labelled primer for $5 \mathrm{~min}$ at $65{ }^{\circ} \mathrm{C}$ in a total volume of $10 \mu \mathrm{l}$. Two microlitres of a $10 \mathrm{mM}$ dNTP mix, $1 \mu \mathrm{l}$ $0.1 \mathrm{M} \mathrm{DTT}, 4 \mu \mathrm{l} 5 \times \mathrm{cDNA}$ buffer, $2 \mu \mathrm{l}$ water and 15 units Thermoscript reverse transcriptase (Invitrogen) were added to each sample and the tubes were incubated at $50{ }^{\circ} \mathrm{C}$ for $45 \mathrm{~min}$. After heating the samples to $85^{\circ} \mathrm{C}$ for $5 \mathrm{~min}$, phenol/chloroform extraction and ethanol precipitation were performed and the pellets were resuspended in $5 \mu$ l water together with $5 \mu \mathrm{l}$ stop solution (T7sequencing kit). A sequencing ladder was generated using the T7sequencing kit (USB) following the instructions of the manufacturer. In brief, $2 \mu \mathrm{g}$ plasmid DNA derived from pJR10::83 were denatured with $2 \mathrm{M} \mathrm{NaOH}$, ethanol precipitated and resuspended in $10 \mu \mathrm{l}$ water. Primer Seqlac (10 pmol in $2 \mu \mathrm{l})$ and $2 \mu \mathrm{l}$ annealing buffer were added and incubated for $5 \mathrm{~min}$ at $65{ }^{\circ} \mathrm{C}$ followed by $10 \mathrm{~min}$ at $37^{\circ} \mathrm{C}$ and $5 \mathrm{~min}$ at room temperature. Then $3 \mu \mathrm{l}$ labelling mix, $1 \mu \mathrm{l}\left[\alpha_{-}{ }^{32} \mathrm{P}\right] \mathrm{dCTP}$ and $2 \mu \mathrm{l}$ diluted T7-DNA polymerase were added. After $5 \mathrm{~min}$ incubation at room temperature, $4.5 \mu \mathrm{l}$ aliquots were added to $2.5 \mu \mathrm{l}$ of the $\mathrm{A}, \mathrm{C}, \mathrm{G}$ and $\mathrm{T}$ termination mixes, respectively. The reaction was stopped after $5 \mathrm{~min}$ by the addition of $5 \mu \mathrm{l}$ stop solution, and loaded next to the primer-extended samples on a denaturing sequencing gel $(6 \%$ acrylamide: bis-acrylamide 19:1, $7 \mathrm{M}$ urea, $0.9 \times \mathrm{TBE})$. The bands were visualized on storage phosphor screens using a Typhoon imager (GE-Healthcare).

\section{RESULTS}

\section{Identification of toxic phage ORFs in M. smegmatis}

As shown previously, the L5 sequence spanning bp 48750 to bp 46034, which covers ORF83 to ORF77, does not produce transformants when introduced into $M$. smegmatis mc ${ }^{2} 155$ (Donnelly-Wu et al., 1993). We could confirm this finding using the construct pJR5::8377 (Fig. 2), which could not be transformed into $M$. smegmatis $\mathrm{mc}^{2} 155$. We then deleted ORF83 from this construct, leading to the plasmid pJR5::8277, which was transformable, giving relatively small $M$. smegmatis colonies on 7H10 agar after 5 days incubation at $37^{\circ} \mathrm{C}$. When transformants were subcultured on $7 \mathrm{H} 10$ agar containing $0.1 \%$ acetamide there was no visible growth, suggesting that the phenotype of clones carrying pJR5::8277 is due to one or more inhibitory ORFs within the phage sequence spanning ORF82 to ORF77. One could also assume that in pJR5::8277 these toxic ORFs are decoupled from a promoter sequence upstream of ORF82 (Fig. 2). To define 


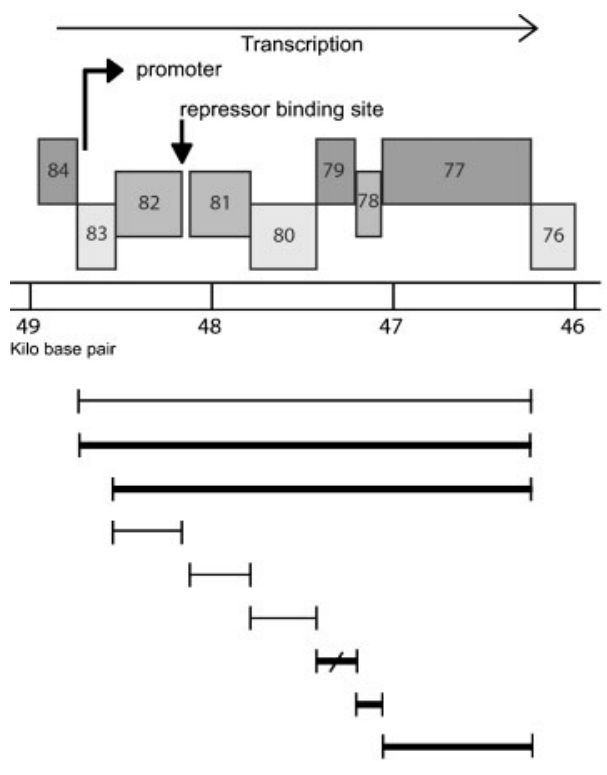

Transformed in E. coli

Fig. 2. Recombinant plasmids created in this study. The upper part illustrates L5 ORFs of the right arm, which predominantly carries early genes. Bold lines indicate inserts with growthinhibitory properties when clones are grown in the presence of acetamide. pJR5 : : 8377 does not produce transformants in $M$. smegmatis whereas pJR5::8277 does, leading to the assumption that ORF83 contains a putative promoter sequence. pJR6::8377 allows transformation since this construct constitutively expresses the phage repressor gp71. pJR5 : : 79/A expresses the $\mathrm{N}$-terminal putative signal peptide of ORF79 and pJR5 : : 79/B the remaining $\mathrm{C}$-terminal part. In contrast to pJR5::79, these constructs lead to wild-type colony morphology when induced.

the exact ORF responsible for toxicity, six plasmids were designed, individually carrying ORF82, 81, 80, 79, 78 or 77 under control of the acetamidase promoter (Fig. 2). Using these plasmids we were able to show that gp77, gp78 and gp79 possess growth-inhibitory properties, with gp77 being the most potent (Fig. 3a, b).

Uninduced $M$. smegmatis clones containing pJR5::77 clearly showed smaller colonies on 7H10 agar compared to the other plasmid-carrying clones (Fig. 3a). This is most likely due to the expression of minute amounts of gp77 from an incompletely repressed acetamidase promoter. This incomplete silencing of the acetamidase promoter in pSD24 was also shown in the ONPG assay as described further below.

\section{Acetamide titration and survival curves of clones carrying toxic phage ORFs}

To identify the minimum amount of acetamide necessary for the growth-inhibitory effect, individual clones were grown to an $\mathrm{OD}_{600}$ of 0.1 and acetamide titration was performed. Here, acetamide concentrations as low as $0.01 \%$ were sufficient to inhibit the growth of clones containing pJR5 : : 77, pJR5:: 78 and pJR5 : : 79. The growth inhibition was directly proportional to the concentration of acetamide as determined by $\mathrm{OD}_{600}$ measurements after $12 \mathrm{~h}$ in a shaking incubator at $37{ }^{\circ} \mathrm{C}$ (Fig. 4a). The $M$. smegmatis clone carrying pJR7, which expresses LacZ under control of the acetamidase promoter, was used as a control.

To further study the effects of L5 toxic gene expression in M. smegmatis (bacteriostatic vs bacteriocidal) survival curves of induced $(0.05 \%$ acetamide $)$ and uninduced clones grown in $7 \mathrm{H} 9$ broth were created by plating serial dilutions every $6 \mathrm{~h}$ for a period of $18 \mathrm{~h}$. Expression of gp77 led to a bacteriostatic effect on M. smegmatis, with growth arrest for all time points examined and recovery of the bacteria after plating on 7H10 agar without acetamide for enumerating c.f.u. (Fig. 4c). Expression of gp78 and gp79 allowed growth of $M$. smegmatis, although at a significantly lower rate compared to the uninduced clones, gp78 being the least potent inhibitor.

\section{Expression of gp79 alters cell morphology of M. smegmatis}

Growing induced M. smegmatis clones carrying pJR5 : : 79 to stationary phase in liquid medium led to the development of non-homogeneous turbidity and the formation of small clumps despite the fact that Tween 80 was added to the medium (Fig. 3b). When grown on solid medium containing $0.1 \%$ acetamide these clones had a rough surface morphology compared to that of wild-type M. smegmatis. Fluorescence microscopy of induced pJR5 : : 79 clones showed filamentation, with most of the bacteria being more than three times longer than uninduced clones and M. smegmatis expressing LacZ, indicating impaired cell division (Fig. 3c). Interestingly, some pJR5::79-bearing cells showed a branching phenotype (Fig. 3c).

\section{Activity of phage ORFs in E. coli}

Since some toxic proteins of bacteriophages infecting Gram-positive bacteria show a growth-inhibitory effect on $E$. coli, we were interested in the phenotype of $E$. coli DH5 $\alpha$ clones expressing L5 proteins encoded by ORF79 to ORF77. In contrast to pSD24 or pJR5, the expression vector pQE80 is tightly regulated, allowing normal growth of uninduced clones. Growth of clones carrying pQE80::77, pQE80::78 and pQE80::79 in the presence of IPTG was clearly inhibited (Fig. $4 \mathrm{~b}, \mathrm{~d}$ ). With respect to gp79, the effect of expression in E. coli was different from that observed in M. smegmatis. Expression of gp79 is clearly 
(a)

(b)
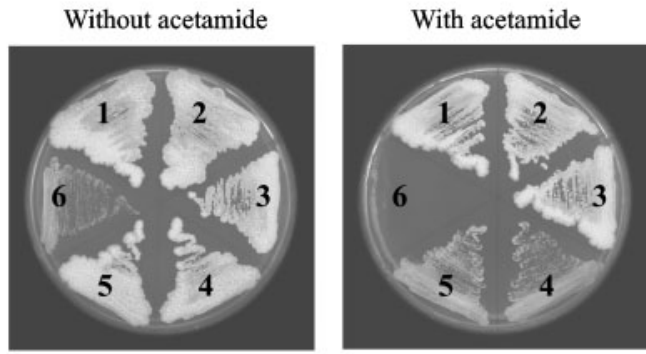

acetamide

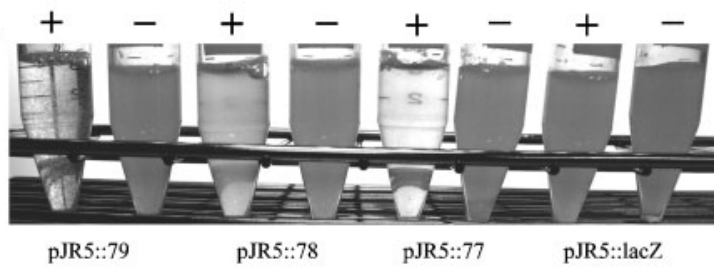

(c)

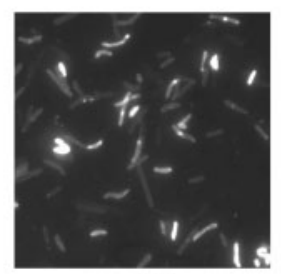

pJR7

(with acetamide)

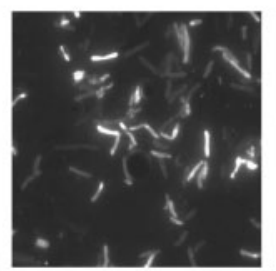

pJR5::79

(without acetamide)

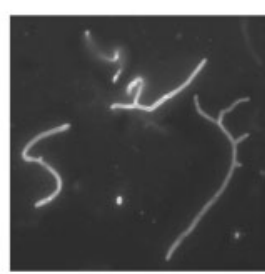

pJR::79 (with acetamide)
Fig. 3. (a) Recombinant plasmids carrying ORF82 to ORF77 (16) under control of the acetamidase promoter cloned into $M$. smegmatis $\mathrm{mc}^{2} 155$ and subcultured with or without the presence of inducing agent. (b) M. smegmatis clones grown to stationary phase in Middlebrook $7 \mathrm{H} 9$ medium plus Tween 80 with or without acetamide $(0.05 \%)$. Expression of gp77, gp78 and 79 is growth inhibitory; the presence of gp79 leads to clumping. (c) Fluorescence microscopy of FITC-stained pJR5::79 clones grown with or without acetamide $(0.05 \%)$; a clone expressing LacZ was used as a control. Expression of gp79 leads to elongation of the individual bacteria and a branching phenotype (photographs taken at $\times 1000$ magnification, reproduced here at $\times 650)$.

bactericidal to E. coli, with almost a $2 \log$ loss of viable cells after $3 \mathrm{~h}$ growth in the presence of $0.1 \mathrm{mM}$ IPTG (Fig. $4 \mathrm{~d}$ ). The expression of gp77 in E. coli led to a bacteriostatic effect whereas the expression of gp78 resulted in a significantly lower growth rate (Fig. $4 \mathrm{~b}, \mathrm{~d}$ ).

\section{Co-expression of the $\mathbf{L} 5$ repressor allows transformation of ORF83 to ORF77}

In contrast to pJR5::8377, which does not transform competent $M$. smegmatis cells, electroporation of pJR6:: 8377, which constitutively expresses the L5 repressor gp71, resulted in approximately $2.4 \times 10^{4}$ transformants per $\mu \mathrm{g}$ plasmid DNA. Interestingly, the colonies obtained varied widely in colony size and morphology. Subcultures of these clones on media containing acetamide led to growth inhibition, showing that the extremely strong acetamidase promoter may overcome transcriptional silencing of the L5 repressor (data not shown).

\section{ORF83 contains a putative promoter that is functional in $M$. smegmatis but not in $E$. coli}

Transformability of pJR5:: 8277 but not of pJR5::8377 indicates the presence of a positive regulatory element within ORF83, which possibly induces the transcription of the three cytotoxic genes. To confirm this hypothesis two new mycobacterial promoter-probe vectors were created using pSD24 as a backbone. The insertion of lac $Z$ from pMC1871 downstream of the acetamidase promoter led to pJR7. This construct allows the insertion of candidate promoter sequences between the acetamidase promoter and the lacZ gene using the single BamHI site (Fig. 1).pJR7 grown without acetamide shows a relatively low but measurable $\beta$-galactosidase activity whereas addition of acetamide to the medium results in an extremely high activity (Fig. 5a). This is consistent with recently published data indicating that the acetamidase promoter in pSD24 is about three times stronger than the mycobacterial hsp60 promoter (Daugelat et al., 2003). Induced pJR7 was used as a positive control in subsequent experiments for the evaluation of the promoter activity of L5 genes. The presence of a functional promoter inserted between the acetamidase promoter and $l a c Z$ gene results in an increased $\beta$-galactosidase activity compared to uninduced clones carrying pJR7.

To abolish any background activity of the acetamidase promoter the complete element was excised, resulting in pJR10 (Fig. 1). This construct had no detectable $\beta$ galactosidase activity, like wild-type M. smegmatis $\mathrm{mc}^{2} 155$.

The insertion of ORF83 together with a small part of the 5 '-end of ORF82 into the linearized promoter-probe vectors (leading to a fusion protein of gp82 and LacZ) gave pJR7::83 and pJR10::83 (Fig. 5a). M. smegmatis transformants harbouring these plasmids showed an increase of $\beta$-galactosidase activity compared to clones containing pJR10 and uninduced pJR7 (Fig. 5a). These constructs mimic the situation seen in pJR5::8377, with the toxic ORFs being exchanged for the lac $Z$ gene. Compared to fully induced pJR7, promoter activity was about three times lower in (uninduced) pJR7::83 and about four times lower in PJR10::83, suggesting that the promoter within ORF83 is of moderate strength (Fig. 5a).

Uninduced pJR7 had measurable activity, indicating incomplete suppression of the acetamidase promoter in pSD24 and related constructs (Fig. 5a). This explains the slower growth of uninduced clones containing pJR5 :: 77 .

Interestingly, E. coli DH5 $\alpha$ containing pJR10::83 (which has shuttle-vector properties) or pMC1871::83, an E. coli promoter-probe vector carrying ORF83, did not show any 
(a)

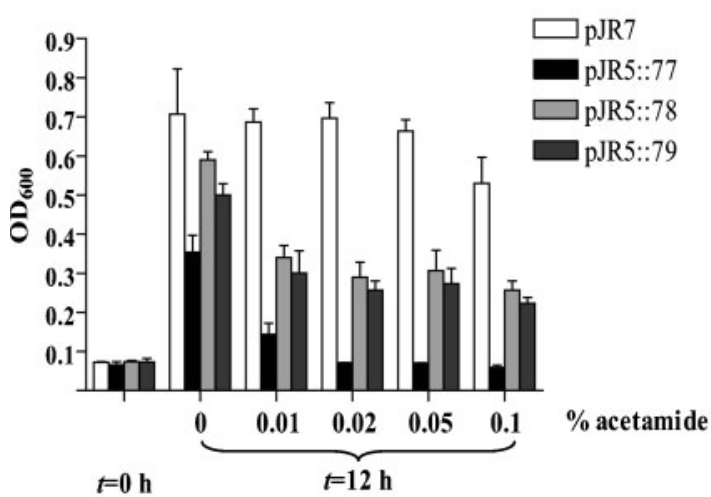

(c)

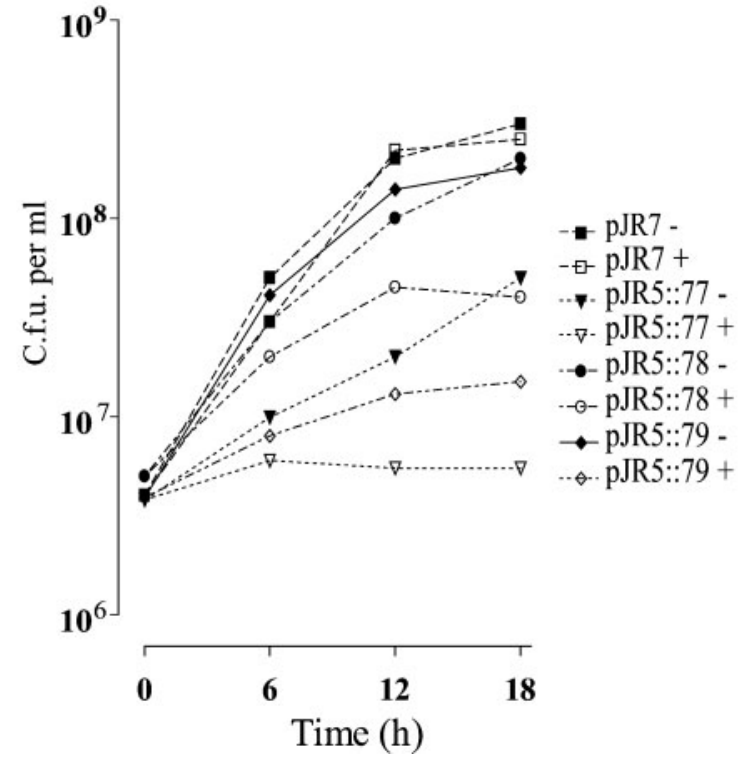

(b)

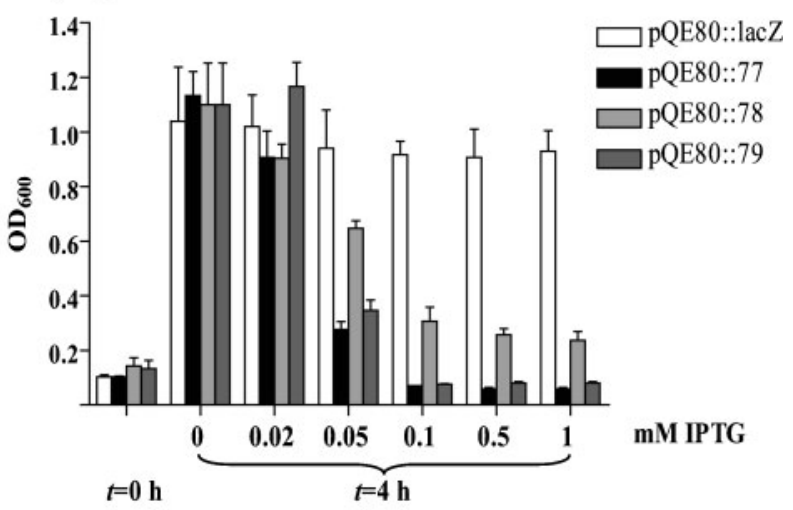

(d)

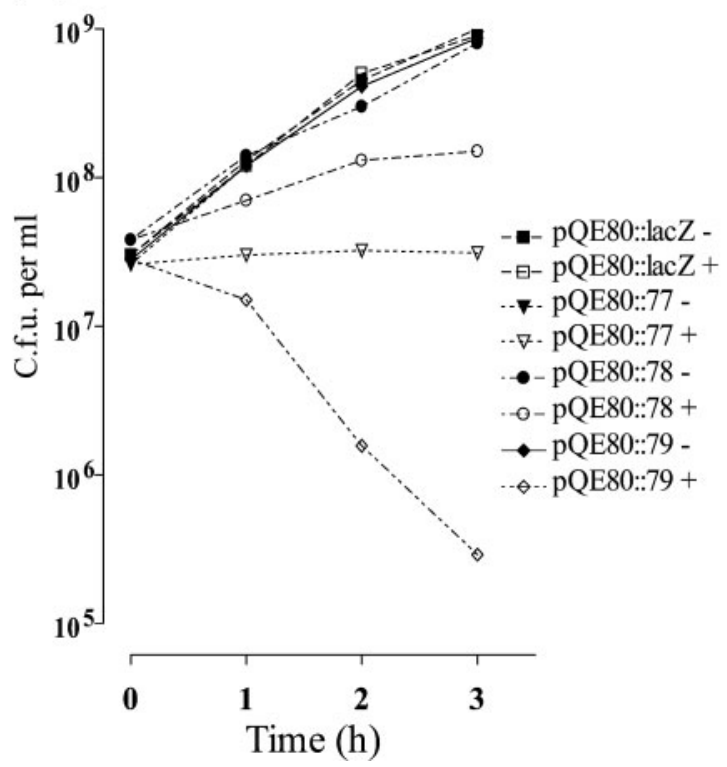

Fig. 4. Different clones expressing toxic phage proteins or LacZ as a control. (a) M. smegmatis clones were grown to early exponential phase and different concentrations of acetamide were added followed by measurement of the $\mathrm{OD}_{600}$ after $12 \mathrm{~h}$. (b) E. coli $\mathrm{DH} 5 \alpha$ clones were grown for $4 \mathrm{~h}$ in the presence of different molarities of IPTG followed by measurement of the OD 600 . Relatively small amounts of inducing agents are needed for growth inhibition, which shows dose dependence. (c) Survival curves of $M$. smegmatis clones grown in $7 \mathrm{H} 9$ medium with $0.05 \%$ acetamide $(+)$ or without acetamide (-). (d) Survival curves of $E$. coli $\mathrm{DH} 5 \alpha$ clones grown in the presence of $0.1 \mathrm{mM} \mathrm{IPTG}(+)$ or without IPTG (-).

$\beta$-galactosidase activity in the ONPG assay (data not shown). This indicates that promoter activity within ORF83 is restricted to mycobacteria.

\section{Detection of the transcriptional start site within ORF83}

Primer extension analysis was performed on M. smegmatis RNA containing either pJR10::83 or pJR10 as a negative control. As shown in Fig. 5(b), a transcriptional start point was detected mapping to position 37 of ORF83. A putative core promoter sequence with -35 (GTGACC) and -10 (TCAAAT) hexamers is situated upstream of this position. The -35 site is homologous with the -35 site of promoter P4 of mycobacteriophage L1, a closely related homoimmune phage of L5 (Chattopadhyay et al., 2003). The experiment was performed with three individual sets of total RNA, which showed identical results. 
(a)

pJR7

pJR10

pJR10::83

pJR7::83

pJR7::82

pJR8::83

pJR7::8382

pJR8::8382

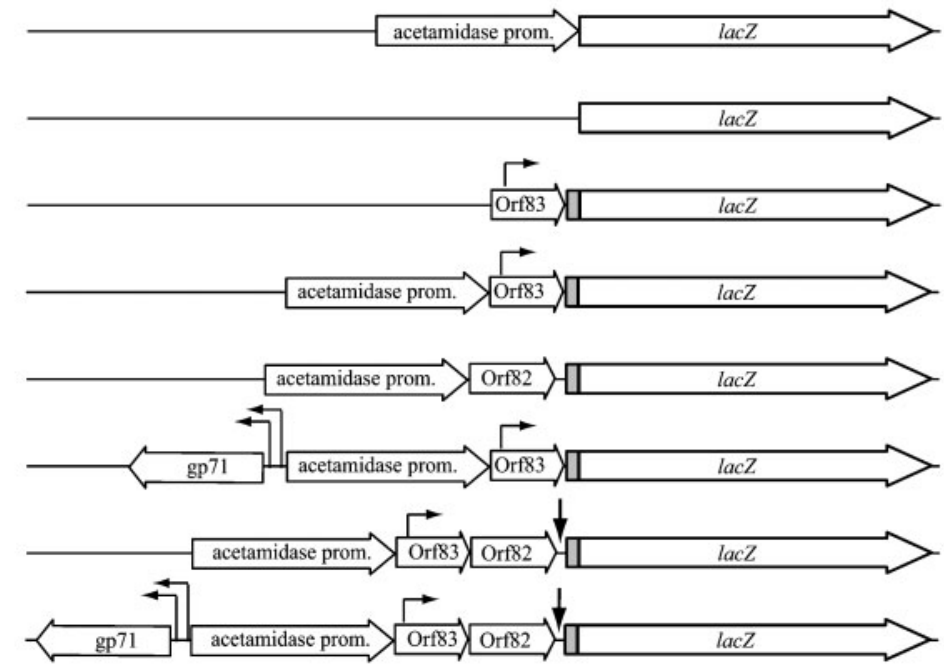

$\beta$-Galactosidase activity (Miller units)

without acetamide / with acetamide
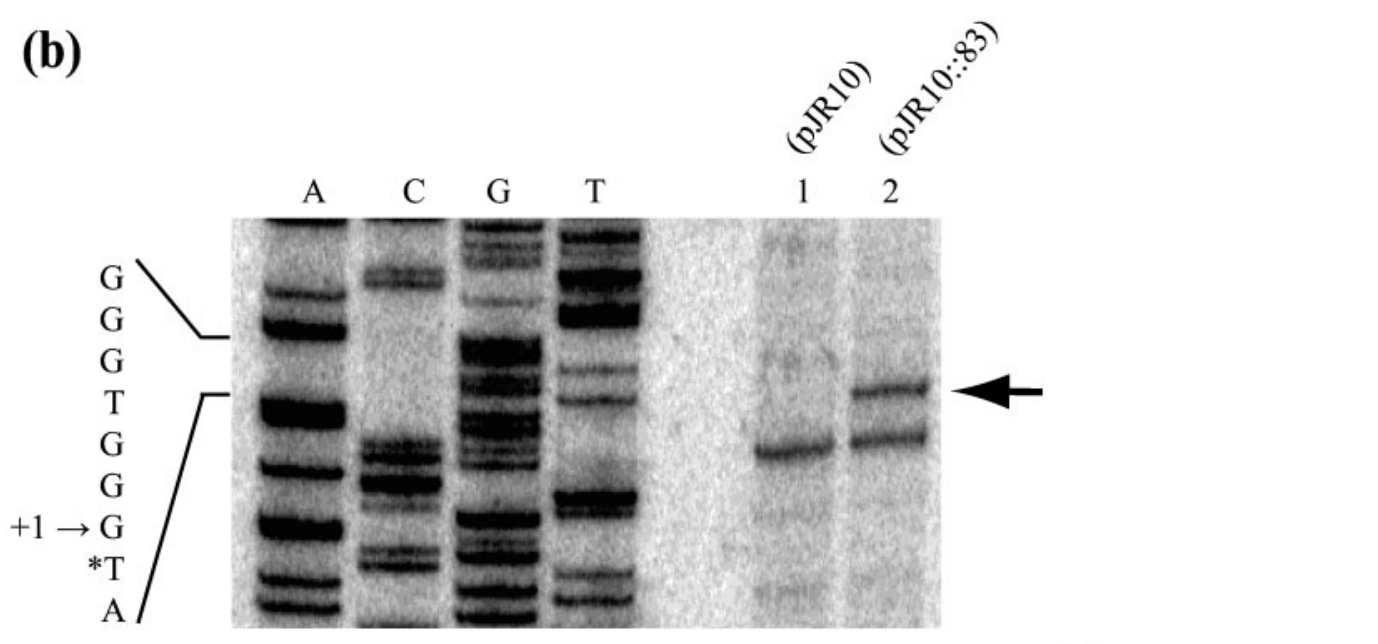

ORF83

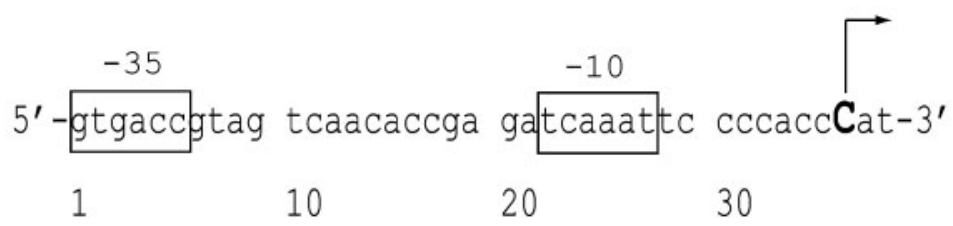

Fig. 5. (a) $\beta$-Galactosidase assay of $M$. smegmatis with different plasmid constructs carrying the lac $Z$ gene. The top two lines show results for cells carrying the new promoter-probe vectors pJR7 and pJR10 grown with and without acetamide (0.1\%). Induced pJR7 functions as a positive control. Uninduced pJR7 shows some activity whereas deletion of the promoter element (pJR10) leads to no detectable $\beta$-galactosidase activity, indicating leakiness of the acetamidase promoter in pSD24. Constructs carrying ORF83 upstream of the lacZ gene show promoter activity within this ORF. Only the presence of both the repressor gp71 and the repressor binding site $(\downarrow)$ between ORF82 and ORF81 (pJR8::8382) leads to a 10-fold reduction of promoter activity, which can be overcome by the strong acetamidase promoter. (b) Primer extension analysis. Lanes A, C, G, T, sequencing ladder generated with pJR10 :: 83 DNA. Lanes 1 and 2, primer extension products obtained with RNA isolated from M. smegmatis clones harbouring pJR10 (promoterless control) and pJR10::83, respectively. A single band was detected matching position $38\left(^{*}\right)$ of the sequencing ladder and position 37 of ORF83 (one base upstream due to use of $\left[\alpha^{-}{ }^{32} \mathrm{P}\right] \mathrm{dCTP}$ for the sequencing ladder and the end-labelled primer for the primer extension experiment). The two lower bands observed in lanes 1 and 2 are presumably non-specific cDNA products. The lower part of the figure shows the DNA sequence of ORF83, with the transcriptional start site at position 37 and putative hexameric -35 and -10 sites indicated by boxes. 


\section{Transcriptional regulation of the putative promoter is achieved through repressor binding between ORF82 and ORF81}

Lysogeny of mycobacteriophage L5 is achieved through binding of the repressor gp71 to asymmetric DNA sites with the consensus sequence GGTGG(C/A)TGTCAAG, which are situated either within some promoter sites or downstream of promoters (Brown et al., 1997). The binding sites within promoters represent true operators that negatively regulate transcription initiation whereas the downstream ones act as stoperators inhibiting transcription elongation, leading to a downregulation of gene expression (Brown et al., 1997). One such stoperator is situated downstream of ORF83 between the stop codon of ORF82 and the start codon of ORF81 (Fig. 2). To show that gp71 and its binding site have a regulatory influence on the promoter element in ORF83, the repressor gene 71 was cloned into the promoter-probe plasmid pJR7, leading to pJR8 (Fig. 1). Insertion of ORF83 upstream of the lacZ gene of $\mathrm{pJR} 8$ led to similar $\beta$-galactosidase activity as seen for pJR7::83 in the ONPG assay whereas integration of ORF83 together with the intergenic region of 82 and 81 (pJR8::8382) led to a 10 -fold reduction of $\beta$-galactosidase activity (Fig. 5a). Addition of acetamide to the medium abolished gp71 activity. A single stoperator site downstream of the extremely strong acetamidase promoter seems to have only a slight negative regulatory effect on promoter activity and thus may not be detectable in this assay.

\section{DISCUSSION}

In this study we have shown that the temperate mycobacteriophage L5 produces toxic proteins leading to growth inhibition of the natural host M. smegmatis. The ORFs encoding the toxic gene products are located in the right arm of the phage genome, which primarily codes for regulatory proteins such as DNA helicase, primase and the repressor (gp71). The finding that a temperate phage expresses early toxic proteins is remarkable and difficult to interpret, especially since the closest relative of L5, mycobacteriophage D29, a lytic phage with high sequence similarity and a shared host range, lacks these early genes and the repressor gene. The fact that the promoter region upstream of the toxic proteins is only of moderate strength does not necessarily mean weak transcription and expression of downstream ORFs since there is at least one additional promoter $\left(\mathrm{P}_{\text {left }}\right)$ upstream of ORF83 (Nesbit et al., 1995). Thus tight regulation of these early gene products is a prerequisite to achieve successful L5 lysogeny. Our co-expression results are in favour of the explanation that transcriptional silencing of the downstream genes during lysogeny is exerted by the phage repressor gp71. These results emphasize the major role of gp71 for globally silencing prophage gene expression, a crucial mechanism for a temperate virus. However, co-expression of gp71 only led to suppression of promoter activity to values just above the background activity of the leaky acetamidase promoter but not to complete silencing of expression. Also, $M$. smegmatis clones carrying pJR6::8377, a plasmid containing the repressor gene, the putative promoter sequence in ORF83, the repressor-binding site and the downstream toxic ORFs, showed variable colony size and morphology. This may reflect unstable expression or a variable half-life of the repressor protein, which could be one explanation for the high rate of L5 infections which lead to lytic growth $(80 \%)$ rather than lysogeny (Sarkis et al., 1995). However, it is not clear how the promoter within ORF83 is regulated in true L5 lysogeny. In contrast to $\mathrm{P}_{\text {left }}$, a promoter upstream of ORF88 that has a gp71-binding site reaching into its -35 sequence controlling the initiation of transcription, there are no such sites in ORF83 (Brown et al., 1997). There may be other, so far undescribed regulatory genes in L5, not present in our plasmid construct, which control transcription during lysogeny.

Although the amino acid sequences of gp77, gp78 and gp79 do not show any sequence homologies with gene products of phages infecting E. coli, we showed that these L5 proteins are inhibitory to the growth of this Gram-negative bacterium. This is not unprecedented since other phagederived shut-off genes such as e3 of phage SPO1, a bacteriophage infecting the Gram-positive Bacillus subtilis, also lead to growth inhibition when expressed in E. coli (Wei \& Stewart, 1993).

The distinct interaction of the three proteins with the host remains unclear. The predicted $28.2 \mathrm{kDa}$ protein encoded by ORF77, which possesses the strongest activity in $M$. smegmatis, does not contain an excess of positively charged amino acids and we were unable to identify any DNAbinding motifs such as helix-turn-helix structures enabling DNA-binding activity. Membrane association of gp77 is unlikely since there is a lack of hydrophobic regions, signal peptides or predicted transmembrane helices. Using pBLAST to compare the amino acid sequence of gp77 with the available database revealed sequence homology not only to other mycobacteriophages but also to hypothetical proteins of actinomycetes such as Frankia spp., Streptomyces spp. and Mycobacterium spp. (Table 2). This indicates that L5 or an L5-related phage may have acquired gp77 from a former host by illegitimate recombination. The toxic protein may have been retained, conferring a selective advantage upon the host.

The N-terminal segment of gp79 (amino acids 1-41) shares sequence similarity with the signal peptide of the D-alanylD-alanine carboxypeptidase of Bacillus licheniformis. This enzyme removes C-terminal D-alanyl residues from sugarpeptide cell-wall precursors and is also a penicillin-binding protein (PBP). It is synthesized with a hydrophobic signal peptide to target it to the cell wall. In most bacterial species, including mycobacteria, PBPs are essential for the biogenesis of the peptidoglycan layer of the cell wall. Knockout of PBP-A in M. smegmatis results in hindered growth and defective cell septation and division (Dasgupta 
Table 2. Molecular mass of gp77, gp78 and gp79, and p-BLAST results

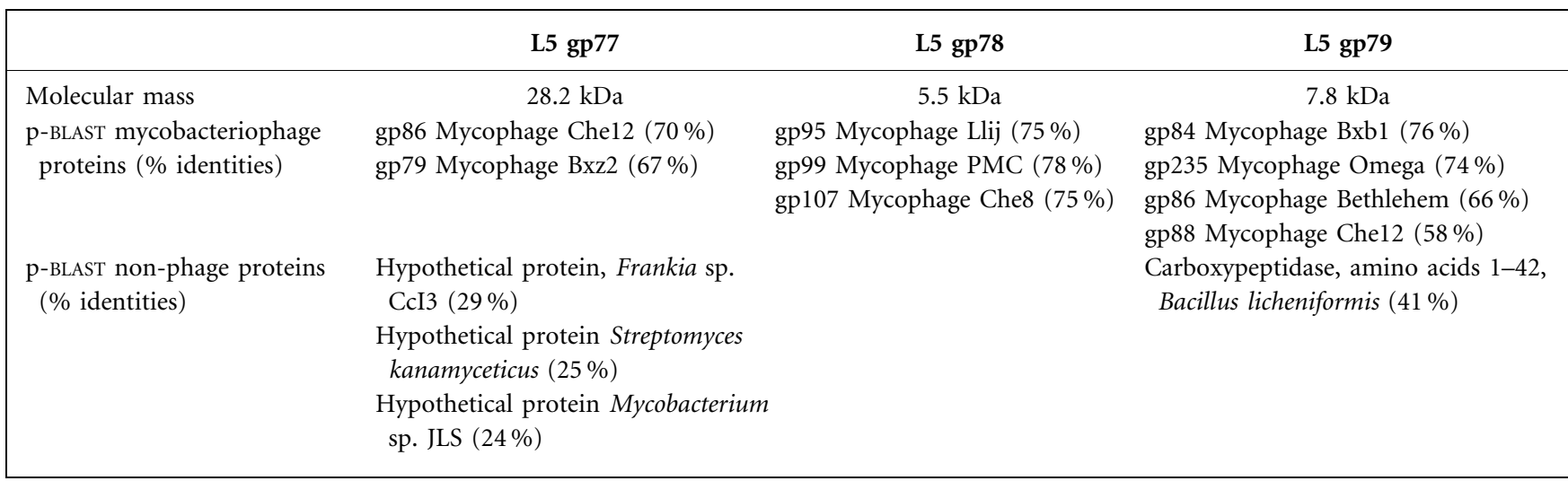

et al., 2006). PBPs are highly conserved across slow- and fast-growing species and interact with several other cellwall-associated proteins such as FtsZ and FtsW. These proteins form a structure called Z-ring, a cytoskeletal scaffold mediating cell division (Datta et al., 2006). Alteration of Z-ring proteins also leads to growth inhibition and impaired cell division in both E. coli and M. smegmatis (Belhumeur \& Drapeau, 1984; Dziadek et al., 2003). Furthermore, simultaneous deletion of several lowmolecular-mass PBPs of E. coli leads to elongation and branching of individual mutants (Nelson \& Young, 2000). This study presents evidence that gp79 interferes with the cell membrane or cell-wall synthesis of M. smegmatis. First, colonies of clones carrying pJR5::79 grown for several days on $7 \mathrm{H} 10$ agar containing $0.1 \%$ acetamide have a rough surface compared to the smooth phenotype of wildtype $M$. smegmatis and clones expressing other L5 proteins. Second, the same clones grown in $7 \mathrm{H} 9$ broth containing acetamide form relatively large clumps even in the presence of Tween 80, again in contrast to wild-type M. smegmatis (Fig. 3b). Third, microscopy of induced clones shows elongated rods compared to the uninduced control, and in some bacterial cells branching can be observed, indicating that gp79 interferes with cell septation (Fig. 3c).

Expression of only the N-terminal hydrophobic part (pJR5::79A, Fig. 2) or the C-terminal part (pJR5::79B, Fig. 2) of gp79 eliminates the toxic effect (data not shown). This indicates that the complete polypeptide is necessary for proper function despite the fact that there is a predicted cleavage site between the alanine at position 34 and the aspartic acid at position 35 .

In addition to the fact that PBP knockout mutants in several bacterial species show a similar phenotype, the homology of the hydrophobic N-terminal part of gp79 to a PBP signal peptide may indicate an interaction of gp79 with proteins or metabolites involved in the peptidoglycan synthesis of M. smegmatis.

The synthesis of the outer cell envelope lipids seems not to be affected by gp79 since their analysis by TLC revealed no difference between induced and uninduced M. smegmatis clones (data not shown).

The reason for L5 interfering with the cell wall of its host in the early stage of phage propagation is not clear. The genes responsible for lysis of the cell wall after completion of the lytic cycle are situated in the far left arm of the phage genome.

The smallest and least active of the toxic proteins described is gp78. This polypeptide, with a predicted molecular mass of $5.5 \mathrm{kDa}$, has detectable sequence homology only to other mycobacteriophage-derived proteins (Table 2), leaving little option for the interpretation of its function.

The data presented here address the intriguing question of the mechanism by which mycobacteriophages 'take over' the host machinery during early lytic growth to replicate to higher levels or to evade host defence. With gp77 and gp79, neighbouring L5 proteins have been described that interfere with the host biogenesis, most likely through two completely different mechanisms.

A potential application of the inducible expression of toxic phage proteins could be as counter-selectable markers for the genetic manipulation of mycobacteria. Furthermore, we note that gp71 expression and binding features combined on a single plasmid provide powerful and convenient applications for the controlled expression of foreign genes in mycobacteria.

In view of the large number of mycobacteriophages in the ecosystem and their extraordinarily high diversity, the approach described in this paper may also allow the further identification of proteins targeting important metabolic pathways of mycobacteria. Target identification is a major step in the drug-discovery process (Terstappen \& Reggiani, 2001). With regard to the pathogenic mycobacteria such as Mycobacterium tuberculosis, determination of the interacting partner proteins on the host's side may lead to the identification and exploitation of as yet unknown key proteins of mycobacterial metabolism and biogenesis. 


\section{ACKNOWLEDGEMENTS}

This work was supported by the Bundesministerium für Bildung und Forschung (BMBF) grants \#01 KI 9952 and \#01 KI 0771. We thank Stefan H. E. Kaufmann at the Max Planck Institute for Infection Biology, Berlin, for providing pSD24. Mycobacteriophage L5 was kindly provided by Graham F. Hatfull at the University of Pittsburgh. We also thank Karin Schnetz from the Institute for Genetics of the University of Cologne for her help with the primer extension experiments, Paul Higgins for his assistance with the ONPG assay and for critical reading of the manuscript, and Werner Falk at the University of Regensburg for his valuable technical advice.

\section{REFERENCES}

Belhumeur, P. \& Drapeau, G. R. (1984). Regulation of cell division in Escherichia coli: properties of new ftsZ mutants. Mol Gen Genet 197, 254-260.

Brown, K. L., Sarkis, G. J., Wadsworth, C. \& Hatfull, G. F. (1997). Transcriptional silencing by the mycobacteriophage L5 repressor. EMBO J 16, 5914-5921.

Chattopadhyay, C., Sau, S. \& Mandal, N. C. (2003). Cloning and characterization of the promoters of temperate mycobacteriophage L1. J Biochem Mol Biol 36, 586-592.

Dasgupta, A., Datta, P., Kundu, M. \& Basu, J. (2006). The serine/ threonine kinase PknB of Mycobacterium tuberculosis phosphorylates PBPA, a penicillin-binding protein required for cell division. Microbiology 152, 493-504.

Datta, P., Dasgupta, A., Singh, A. K., Mukherjee, P., Kundu, M. \& Basu, J. (2006). Interaction between FtsW and penicillin-binding protein 3 (PBP3) directs PBP3 to mid-cell, controls cell septation and mediates the formation of a trimeric complex involving FtsZ, FtsW and PBP3 in mycobacteria. Mol Microbiol 62, 1655-1673.

Daugelat, S., Kowall, J., Mattow, J., Bumann, D., Winter, R., Hurwitz, R. \& Kaufmann, S. H. (2003). The RD1 proteins of Mycobacterium tuberculosis: expression in Mycobacterium smegmatis and biochemical characterization. Microbes Infect 5, 1082-1095.

Donnelly-Wu, M. K., Jacobs, W. R., Jr \& Hatfull, G. F. (1993). Superinfection immunity of mycobacteriophage L5: applications for genetic transformation of mycobacteria. Mol Microbiol 7, 407-417.

Dziadek, J., Rutherford, S. A., Madiraju, M. V., Atkinson, M. A. \& Rajagopalan, M. (2003). Conditional expression of Mycobacterium smegmatis fts $Z$, an essential cell division gene. Microbiology 149, 1593-1603.

Fenwick, M. L. \& Clark, J. (1982). Early and delayed shut-off of host protein synthesis in cells infected with herpes simplex virus. $J$ Gen Virol 61, 121-125.

Hatfull, G. F. \& Sarkis, G. J. (1993). DNA sequence, structure and gene expression of mycobacteriophage L5: a phage system for mycobacterial genetics. Mol Microbiol 7, 395-405.

Hatfull, G. F., Pedulla, M. L., Jacobs-Sera, D., Cichon, P. M., Foley, A., Ford, M. E., Gonda, R. M., Houtz, J. M., Hryckowian, A. J. \& other authors (2006). Exploring the mycobacteriophage metaproteome: phage genomics as an educational platform. PLoS Genet 2, e92.
Liu, J., Dehbi, M., Moeck, G., Arhin, F., Bauda, P., Bergeron, D., Callejo, M., Ferretti, V., Ha, N. \& other authors (2004). Antimicrobial drug discovery through bacteriophage genomics. Nat Biotechnol 22, 185-191.

Nelson, D. E. \& Young, K. D. (2000). Penicillin binding protein 5 affects cell diameter, contour, and morphology of Escherichia coli. $J$ Bacteriol 182, 1714-1721.

Nesbit, C. E., Levin, M. E., Donnelly-Wu, M. K. \& Hatfull, G. F. (1995). Transcriptional regulation of repressor synthesis in mycobacteriophage L5. Mol Microbiol 17, 1045-1056.

Pedulla, M. L., Ford, M. E., Houtz, J. M., Karthikeyan, T., Wadsworth, C., Lewis, J. A., Jacobs-Sera, D., Falbo, J., Gross, J. \& other authors (2003). Origins of highly mosaic mycobacteriophage genomes. Cell 113, 171-182.

Robinson, N., Wolke, M., Ernestus, K. \& Plum, G. (2007). A mycobacterial gene involved in synthesis of an outer cell envelope lipid is a key factor in prevention of phagosome maturation. Infect Immun 75, 581-591.

Rybniker, J., Kramme, S. \& Small, P. L. (2006). Host range of 14 mycobacteriophages in Mycobacterium ulcerans and seven other mycobacteria including Mycobacterium tuberculosis - application for identification and susceptibility testing. J Med Microbiol 55, 37-42.

Sambrook, J. \& Russell, D. W. (2001). Molecular Cloning: a Laboratory Manual, 3rd edn. Cold Spring Harbor, NY: Cold Spring Harbor Laboratory.

Sarkis, G. J., Jacobs, W. R., Jr \& Hatfull, G. F. (1995). L5 luciferase reporter mycobacteriophages: a sensitive tool for the detection and assay of live mycobacteria. Mol Microbiol 15, 1055-1067.

Shapira, S. K., Chou, J., Richaud, F. V. \& Casadaban, M. J. (1983). New versatile plasmid vectors for expression of hybrid proteins coded by a cloned gene fused to lac $Z$ gene sequences encoding an enzymatically active carboxy-terminal portion of beta-galactosidase. Gene 25, 71-82.

Sharma, R., Raychaudhuri, S. \& Dasgupta, A. (2004). Nuclear entry of poliovirus protease-polymerase precursor 3CD: implications for host cell transcription shut-off. Virology 320, 195-205.

Snapper, S. B., Melton, R. E., Mustafa, S., Kieser, T. \& Jacobs, W. R., Jr (1990). Isolation and characterization of efficient plasmid transformation mutants of Mycobacterium smegmatis. Mol Microbiol 4, 1911-1919.

Stinear, T. P., Seemann, T., Pidot, S., Frigui, W., Reysset, G., Garnier, T., Meurice, G., Simon, D., Bouchier, C. \& other authors (2007). Reductive evolution and niche adaptation inferred from the genome of Mycobacterium ulcerans, the causative agent of Buruli ulcer. Genome Res 17, 192-200.

Svenson, S. B. \& Karlstrom, O. H. (1976). Bacteriophage T4-induced shut-off of host-specific translation. J Virol 17, 326-334.

Terstappen, G. C. \& Reggiani, A. (2001). In silico research in drug discovery. Trends Pharmacol Sci 22, 23-26.

Wei, P. \& Stewart, C. R. (1993). A cytotoxic early gene of Bacillus subtilis bacteriophage SPO1. J Bacteriol 175, 7887-7900.

Zhang, X. \& Bremer, H. (1995). Control of the Escherichia coli rrnB P1 promoter strength by ppGpp. J Biol Chem 270, 11181-11189.

Edited by: P. R. Herron 